Raymond H. Mak, Brigham and Women's Hospital/Dana-Farber Cancer Institute and Harvard Medical School; William U. Shipley, Jason A. Efstathiou, Donald S. Kaufman, Niall M. Heney, and Anthony L. Zietman, Massachusetts General Hospital and Harvard Medical School, Boston, MA; Daniel Hunt, Radiation Therapy Oncology Group Statistical Center; William J. Tester, Albert Einstein Medical Center, Philadelphia, PA; and Michael P. Hagan Veterans Health Administration, Richmond, VA.

Published online ahead of print at www.jco.org on November 3, 2014.

Supported by Radiation Therapy Oncology Group Grant No. U10 CA21661 and Community Clinical Oncology Program Grant No. U10 CA37422 from the National Cancer Institute.

Presented in part at the 2012 Genitourinary Cancers Symposium, San Francisco, CA, February 2-4, 2012, and the 54th Annual Meeting of the American Society for Radiation Oncology, Boston, MA, October 28-31, 2012

Clinical trial information: NCT00055601, NCT00003930.

The contents of this article are solely the responsibility of the authors and do not necessarily represent the official views of the National Cancer Institute.

Authors' disclosures of potential conflicts of interest are found in the article online at www.jco.org. Author contributions are found at the end of this article.

Corresponding author: Raymond $\mathrm{H}$ Mak, MD, 75 Francis St, ASB1 L2, Department of Radiation Oncology, Brigham and Women's Hospital/DanaFarber Cancer Institute, Boston, MA 02115; e-mail: rmak@|roc.harvard.edu.

() 2014 by American Society of Clinical Oncology

0732-183X/14/3234w-3801w/\$20.00

DOI: $10.1200 / J C O .2014 .57 .5548$

\title{
Long-Term Outcomes in Patients With Muscle-Invasive Bladder Cancer After Selective Bladder-Preserving Combined-Modality Therapy: A Pooled Analysis of Radiation Therapy Oncology Group Protocols 8802, 8903, 9506, 9706, 9906, and 0233
}

Raymond H. Mak, Daniel Hunt, William U. Shipley, Jason A. Efstathiou, William J. Tester, Michael P. Hagan, Donald S. Kaufman, Niall M. Heney, and Anthony L. Zietman

See accompanying editorial on page 3787

$$
\begin{array}{llllllll}
\text { A } & \text { B } & \text { S } & \text { T } & \text { R } & \text { A } & \text { C } & \text { T }
\end{array}
$$

\section{Purpose}

Multiple prospective Radiation Therapy Oncology Group (RTOG) protocols have evaluated bladderpreserving combined-modality therapy (CMT) for muscle-invasive bladder cancer (MIBC), reserving cystectomy for salvage treatment. We performed a pooled analysis of long-term outcomes in patients with MIBC enrolled across multiple studies.

\section{Patients and Methods}

Four hundred sixty-eight patients with MIBC were enrolled onto six RTOG bladder-preservation studies, including five phase II studies (RTOG 8802, 9506, 9706, 9906, and 0233) and one phase III study (RTOG 8903). Overall survival (OS) was estimated using the Kaplan-Meier method, and disease-specific survival (DSS), muscle-invasive and non-muscle-invasive local failure (LF), and distant metastasis (DM) were estimated by the cumulative incidence method.

\section{Results}

The median age of patients was 66 years (range, 34 to 93 years), and clinical T stage was T2 in 61\%, T3 in 35\%, and T4a in 4\% of patients. Complete response to CMT was documented in $69 \%$ of patients. With a median follow-up of 4.3 years among all patients and 7.8 years among survivors $(n=$ 205), the 5- and 10-year OS rates were 57\% and 36\%, respectively, and the 5- and 10-year DSS rates were $71 \%$ and $65 \%$, respectively. The 5 - and 10 -year estimates of muscle-invasive LF, non-muscleinvasive LF, and DM were $13 \%$ and $14 \%, 31 \%$ and $36 \%$, and $31 \%$ and $35 \%$, respectively.

\section{Conclusion}

This pooled analysis of multicenter, prospective RTOG bladder-preserving CMT protocols demonstrates long-term DSS comparable to modern immediate cystectomy studies, for patients with similarly staged MIBC. Given the low incidence of late recurrences with long-term follow-up, CMT can be considered as an alternative to radical cystectomy, especially in elderly patients not well suited for surgery.

\section{J Clin Oncol 32:3801-3809. (c) 2014 by American Society of Clinical Oncology}

\section{INTRODUCTION}

Although radical cystectomy (RC) remains the usual treatment for muscle-invasive bladder cancer (MIBC) in the United States, bladder-preserving treatment strategies have evolved over the past 20 years with continued refinements in radiation therapy (RT), chemotherapy for radiation sensitization, and patient selection that can provide selected patients with an excellent chance for long-term survival with an intact, functioning bladder. The hallmarks of modern bladder-preserving therapy include combined-modality therapy (CMT) with maximal transurethral resection of bladder tumor (TURBT), RT, and concurrent chemotherapy; cystoscopic assessment of response to therapy with prompt salvage RC for nonresponders; and careful follow-up with cystoscopic surveillance and prompt RC for invasive recurrence. Successive Radiation Therapy Oncology Group (RTOG) studies have demonstrated that this bladder-preserving CMT approach in patients presenting with MIBC can 
achieve high rates of complete tumor response, bladder preservation in the majority of patients, and survival rates similar to those seen in contemporary RC series. ${ }^{1-8}$ Contemporary bladder-preserving approaches in patients with clinically staged MIBC can achieve complete response (CR) rates of $60 \%$ to $80 \%$, 5-year disease-specific survival (DSS) rates of $60 \%$ to $70 \%$, and bladder intact survival rates of $40 \%$ to $45 \%$. ${ }^{9,10}$

The long-term, 10-year results of CMT have been reported from several single-institution series including from the Massachusetts General Hospital (MGH; Boston, MA) and University of Erlangen (Erlangen, Germany)..$^{9-12}$ Long-term follow-up of 348 patients with MIBC treated with chemotherapy and RT in 1986 to 2002 at MGH showed 10- and 15-year DSS rates of 59\% and 57\%, respectively, and 10 - and 15 -year OS rates of $35 \%$ and $22 \%$, respectively. The University of Erlangen reported on 415 patients treated from 1982 to 2000 and demonstrated a 10 -year DSS rate of $42 \%$, with $80 \%$ of surviving patients preserving their bladders. Long-term follow-up of CMT in a pooled analysis of patients from RTOG bladder-preservation studies demonstrated a low incidence of late pelvic toxicity in patients retaining their bladder. ${ }^{13}$
However, the long-term outcomes of bladder-preserving CMT for MIBC, including OS, DSS, and long-term bladder-preservation rates, have not been examined in the multi-institutional setting. Here, we report a pooled secondary analysis of the long-term outcomes of patients who received bladder-preserving CMT in six RTOG studies.

\section{PATIENTS AND METHODS}

Data and outcomes of 468 patients with MIBC enrolled onto six RTOG bladder-preservation studies were pooled, including five phase II studies (RTOG 8802, 9506, 9706, 9906, and 0233) and one phase III study (RTOG 8903; Appendix Table A1, online only). Eligibility criteria for these trials have been previously described but included clinical T2-4a stage and excluded patients with biopsy-proven nodal disease or metastatic disease. Trials subsequent to RTOG 8802 and RTOG 8903 excluded patients with hydronephrosis.

\section{RTOG Trials in the Pooled Analysis}

RTOG 8802. This phase II trial enrolled 90 eligible patients from 1988 to 1990 who received two cycles of neoadjuvant methotrexate (30

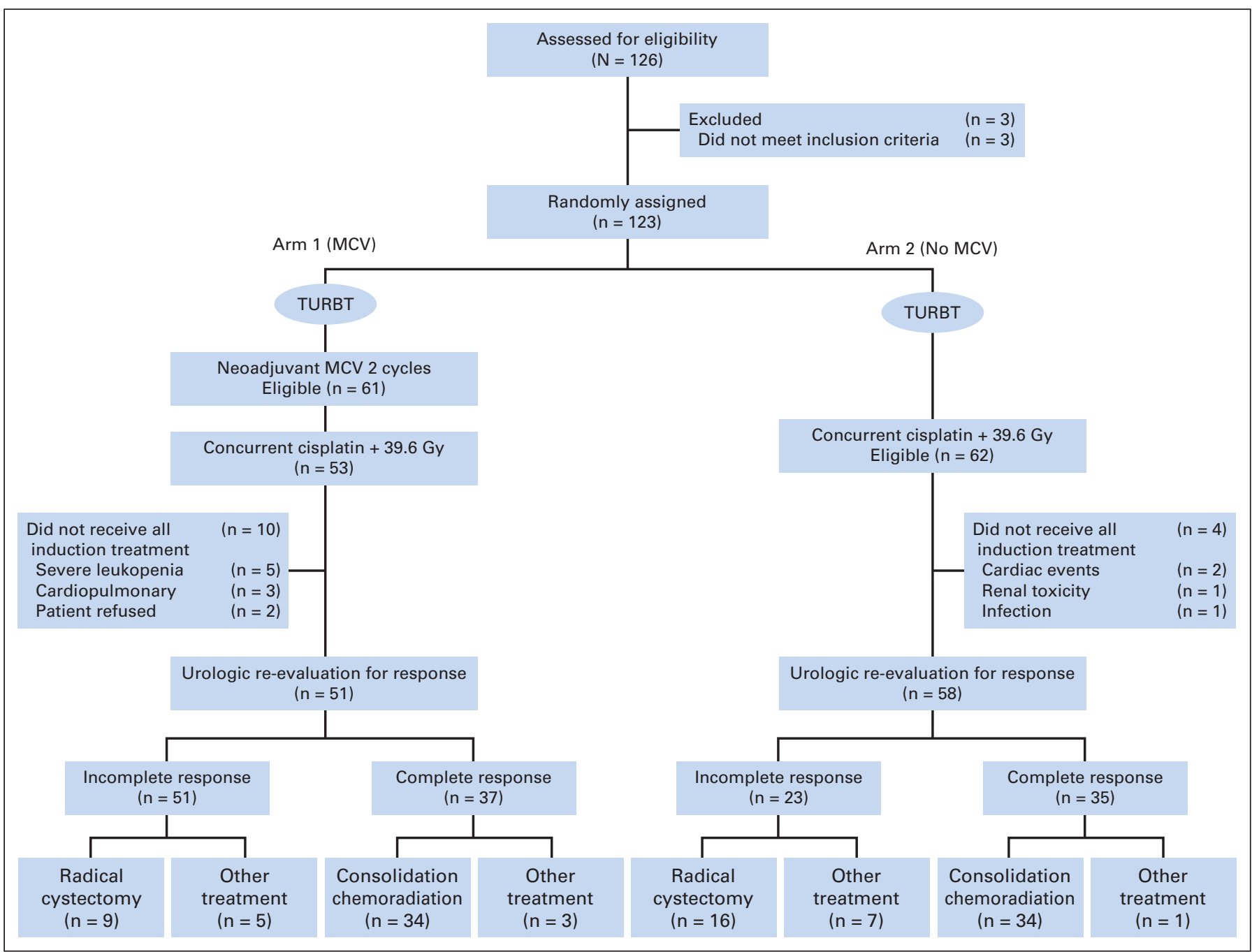

Fig 1. CONSORT diagram of Radiation Therapy Oncology Group protocol 8903. MCV, methotrexate, cisplatin, and vinblastine; TURBT, transurethral resection of bladder tumor. Data adapted. ${ }^{15}$ 


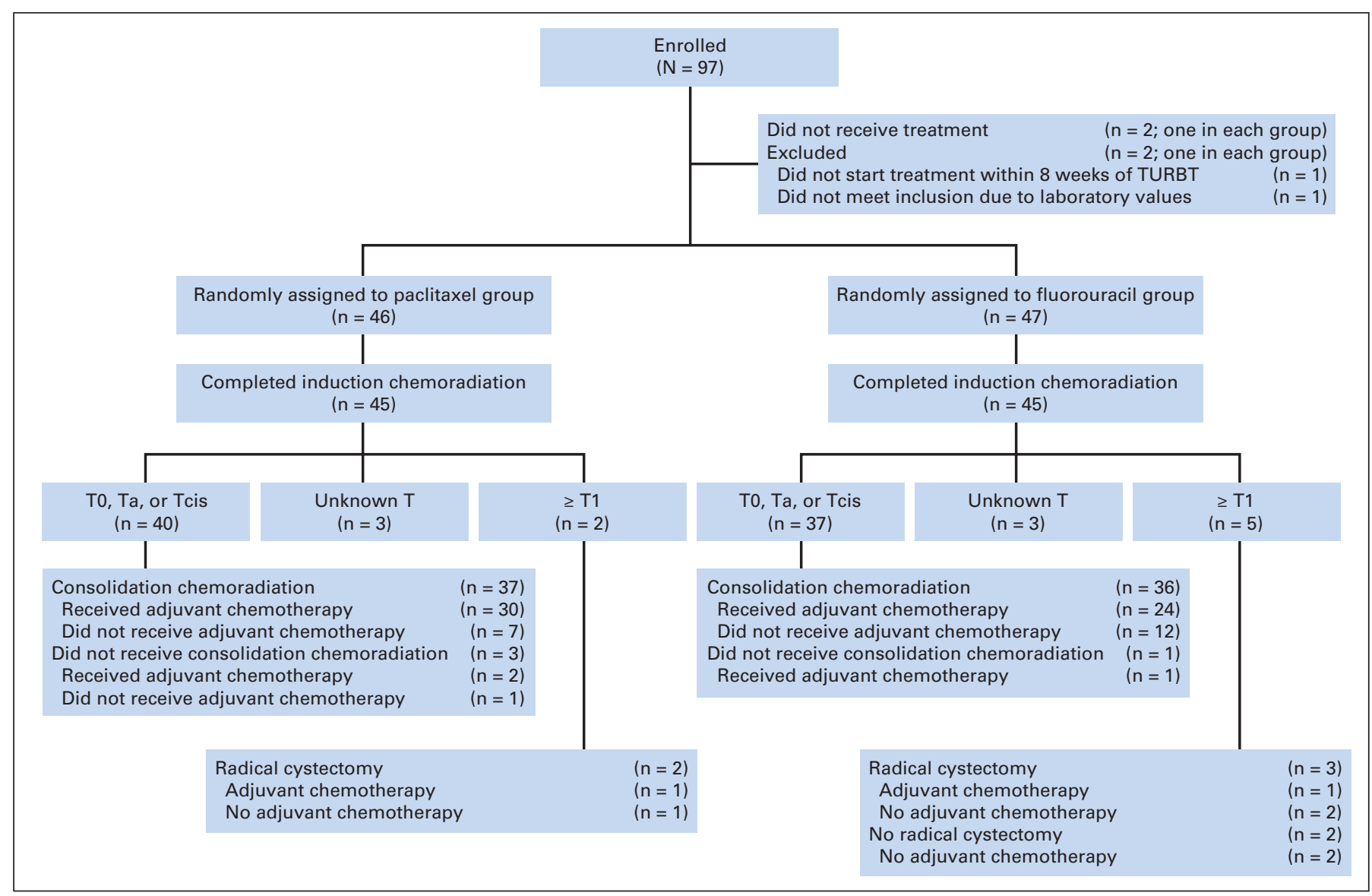

Fig 2. CONSORT diagram of Radiation Therapy Oncology Group protocol 0233. MCV, methotrexate, cisplatin, and vinblastine; TURBT, transurethral resection of bladder tumor. Data adapted. ${ }^{19}$

$\left.\mathrm{mg} / \mathrm{m}^{2}\right)$, cisplatin $\left(70 \mathrm{mg} / \mathrm{m}^{2}\right)$, and vinblastine $\left(3 \mathrm{mg} / \mathrm{m}^{2} ; \mathrm{MCV}\right)$ followed by once-daily RT to 39.6 Gy with concurrent cisplatin $\left(70 \mathrm{mg} / \mathrm{m}^{2}\right.$ every 3 weeks). ${ }^{14}$ The RT consisted of a small pelvic four-field technique (similar for all protocols), encompassing the whole bladder, bladder tumor, prostate (in men), and adjacent pelvic lymph nodes.

RTOG 8903. This phase III trial randomly assigned 123 eligible patients from 1990 to 1993 at 37 centers to two cycles of neoadjuvant MCV versus no $\mathrm{MCV}$, followed by once-daily pelvic RT to $39.6 \mathrm{~Gy}$ and two cycles of concurrent cisplatin $\left(100 \mathrm{mg} / \mathrm{m}^{2}\right.$ every 3 weeks; Fig 1$) .{ }^{15}$ Patients with a CR on rebiopsy after 39.6 Gy or RT received consolidation therapy with 25.2 Gy of RT (total dose, $64.8 \mathrm{~Gy}$ ) and one additional cycle of cisplatin.

RTOG 9506. This phase I/II trial enrolled 34 eligible patients from 1995 to 1997 at 11 institutions. After TURBT, patients received induction accelerated hypofractionated (3 Gy per fraction) twice-daily RT to the pelvis (24 Gy) with concurrent cisplatin $\left(15 \mathrm{mg} / \mathrm{m}^{2}\right.$ on days 1 through 3 and 15 through 17) and fluorouracil (FU; $400 \mathrm{mg} / \mathrm{m}^{2}$ on days 1 through 3 and 15 through 17). Patients with a CR received twice-daily RT ( 2.5 Gy per fraction) to the whole bladder and the bladder tumor (total dose, 44 Gy to bladder and tumor and 24 Gy to pelvic lymph nodes) with concurrent FU/cisplatin. ${ }^{16}$

RTOG 9706. This phase I/II study enrolled 47 eligible patients from 1997 to 1999 at 17 institutions. After TURBT, patients received induction twice-daily RT with 1.8 Gy to the pelvis in the morning and 1.6 Gy to the bladder tumor in the afternoon for 13 days (40.8 Gy to bladder tumor and 21.6 Gy to the pelvis) with concurrent weekly cisplatin $\left(20 \mathrm{mg} / \mathrm{m}^{2}\right.$ first 2 days per week). Patients with a CR on rebiopsy after 41.6 Gy of RT received twice-daily RT (1.5 Gy per fraction) given to each site for 8 days (total dose, 45.6 Gy to pelvis and bladder and 64.8 Gy to bladder tumor) with weekly cisplatin. ${ }^{17}$

RTOG 9906. This phase I/II trial enrolled 81 eligible patients from 1999 to 2002 at 26 institutions. After TURBT, patients underwent induction twice- daily accelerated RT over 13 days, including 1.6 Gy to the pelvis in the morning, and 1.5 Gy to the bladder for the first 5 days $(7.5 \mathrm{~Gy})$ and then $1.5 \mathrm{~Gy}$ to the tumor for the next 8 days $(12.0 \mathrm{~Gy}$ ) in the afternoons (total dose, $20.8 \mathrm{~Gy}$ to pelvis, 28.3 Gy to whole bladder, and $40.3 \mathrm{~Gy}$ to bladder tumor) with concurrent weekly cisplatin $\left(20 \mathrm{mg} / \mathrm{m}^{2} 2\right.$ days a week) and paclitaxel $\left(50 \mathrm{mg} / \mathrm{m}^{2}\right.$ per week). Patients with a CR on rebiopsy after $40.3 \mathrm{~Gy}$ of RT received 1.5-Gy twice-daily pelvic RT to a dose of 24 Gy (total dose, 64.3 Gy to the bladder tumor and 44.8 Gy to the pelvis) with concurrent weekly cisplatin/paclitaxel. Patients then received four cycles of adjuvant cisplatin $\left(70 \mathrm{mg} / \mathrm{m}^{2}\right)$ and gemcitabine $\left(1,000 \mathrm{mg} / \mathrm{m}^{2}\right){ }^{18}$

RTOG 0233. This phase II randomized trial enrolled 93 eligible patients from 2003 to 2007 at 24 institutions (Fig 2). ${ }^{19}$ After TURBT, patients received twice-daily RT, as in RTOG 9906, and were randomly assigned to either concurrent paclitaxel $\left(50 \mathrm{mg} / \mathrm{m}^{2}\right.$ per week $)$ plus cisplatin $\left(15 \mathrm{mg} / \mathrm{m}^{2} 3\right.$ days per week; $\mathrm{n}=46)$ or $\mathrm{FU}\left(500 \mathrm{mg} / \mathrm{m}^{2} 2\right.$ days per alternate week) plus cisplatin ( $\mathrm{n}=$ 47) during the induction and consolidation phases followed by adjuvant gemcitabine/paclitaxel/cisplatin.

\section{Criteria for CR and Follow-Up}

Clinical CR was defined as no tumor palpable on bimanual examination under anesthesia, no tumor visible on cystoscopy, negative tumor site biopsy, and negative urine cytology. Patients with preserved bladders underwent routine active surveillance including cystoscopy, tumor site biopsy, bimanual examination under anesthesia, and urine cytology every 3 months for the first year, and then cystoscopy and cytology every 3 to 4 months during the second year, every 6 months for 3 years, and then annually. Patients with non-muscle-invasive local failure (LF) were promptly considered for intravesical therapy, and patients with a muscleinvasive LF underwent salvage RC. 


\section{End Points}

All end points were measured from the date of study entry (phase II studies) or random assignment (phase III study) to the date of first documented event. For overall survival (OS) and DSS, survival time was measured to date of death (as a result of any cause) or death from disease, respectively. Time to LF was measured to the date of documented tumor recurrence after CR or to the date of 1 day after study entry/random assignment in patients who did not achieve CR. LF after a CR to induction CMT was examined in the following two subcategories: muscle-invasive and non-muscle-invasive LF. Nodal recurrence was defined as documented presence or progression of regional (pelvic) nodes. Time to distant metastasis (DM) was defined by the date of first documented DM. Time to bladder-intact diseasefree survival was defined as time to the earliest of muscle-invasive local recurrence in the bladder, regional pelvic recurrence, DM, bladder cancer-related death, or cystectomy.

\section{Statistical Analyses}

OS and bladder-intact disease-free survival were estimated using the Kaplan-Meier method. ${ }^{20}$ LF, nodal recurrences, DM, and DSS were estimated using cumulative incidence methodology. ${ }^{21}$ Fine and Gray's proportional hazards regression model was performed to identify clinical variables associated with DSS. The following covariates were included in the multiple regression model: age, sex, T stage (T2 $v$ T3/T4a), histology (urothelial carcinoma $v$ other), tumor grade (low grade $v$ high grade), presence of hydronephrosis, and whether the TURBT was visibly complete or not. All statistical comparisons were two-sided, and $P<.05$ was considered statistically significant. SAS software (SAS Institute, Cary, NC) was used for all analyses, except for the Fine and Gray's modeling, which was done in R (R Foundation for Statistical Computing, Vienna, Austria).

\section{RESULTS}

\section{Patients}

From 1988 to 2007, 468 eligible patients were enrolled onto these successive RTOG protocols. Patient and tumor characteristics are listed in Table 1.

\section{Long-Term Outcomes}

A CR to induction chemotherapy and RT occurred in 69\% of patients (321 of 468 patients). With a median follow-up of 4.3 years among all patients and 7.8 years among patients alive at the time of this analysis $(\mathrm{n}=205)$, the 5 - and 10 -year OS rates were $57 \%$ and $36 \%$, respectively (Fig 3A). The 5- and 10-year DSS rates were $71 \%$ and $65 \%$, respectively (Fig 3B; Table 2). Bladder cancer was the cause of death in $24 \%$ of patients who had died by 5 years $(n=191)$. Of the 205 patients alive at 5 years, $80 \%$ had an intact bladder.

The 5- and 10-year LF estimates were $43 \%$ and $48 \%$, respectively, and the majority of LFs were non-muscle-invasive LFs instead of muscle-invasive LF (Table 2). For all patients, including those with and without salvage RC, the 5- and 10-year estimates of nodal recurrence were $13 \%$ and $16 \%$, respectively. The 5- and 10-year DM estimates were $31 \%$ and $35 \%$, respectively. The long-term outcomes by trial are listed in Appendix Table A2 (online only).

\section{Outcomes by Response}

In patients with a CR after induction CMT, DSS was significantly higher than in patients who did not have a CR (5-year DSS, 79\% $v$ 56\%, respectively; 10 -year DSS, 74\% v 47\%, respectively; $P<.001$; Fig $3 \mathrm{E})$. The 5 - and 10 -year OS rates for patients with a CR were $65 \%$ and $43 \%$, respectively, compared with $44 \%$ and $25 \%$, respectively, in patients who did not have a CR.

\begin{tabular}{|c|c|c|}
\hline Characteristic & No. of Patients ( $N=468)$ & $\%$ \\
\hline \multicolumn{3}{|l|}{ Age, years } \\
\hline Median & \multicolumn{2}{|l|}{66} \\
\hline Range & \multicolumn{2}{|l|}{$34-93$} \\
\hline$<70$ & 300 & 64.1 \\
\hline $70-75$ & 88 & 18.8 \\
\hline$\geq 75$ & 80 & 17.1 \\
\hline \multicolumn{3}{|l|}{ Zubrod score } \\
\hline 0 & 417 & 89.1 \\
\hline 1 & 51 & 10.9 \\
\hline \multicolumn{3}{|l|}{ Sex } \\
\hline Male & 386 & 82.5 \\
\hline Female & 82 & 17.5 \\
\hline \multicolumn{3}{|l|}{ Race } \\
\hline White & 358 & 76.5 \\
\hline Hispanic or Latino & 10 & 2.1 \\
\hline Black or African American & 11 & 2.4 \\
\hline Asian & 87 & 18.6 \\
\hline Unknown & 2 & 0.4 \\
\hline \multicolumn{3}{|l|}{ Histology } \\
\hline Urothelial & 439 & 94.2 \\
\hline Squamous & 6 & 1.3 \\
\hline Adenocarcinoma & 5 & 1.1 \\
\hline Other & 16 & 3.4 \\
\hline \multicolumn{3}{|l|}{ Clinical T stage } \\
\hline $\mathrm{T} 2$ & 283 & 60.6 \\
\hline T3 & 1 & 0.2 \\
\hline Т3а & 136 & 29.1 \\
\hline T3b & 29 & 6.2 \\
\hline $\mathrm{T} 4 \mathrm{a}$ & 18 & 3.9 \\
\hline \multicolumn{3}{|l|}{ Hydronephrosis } \\
\hline Yes & 40 & 10.6 \\
\hline No & 337 & 89.4 \\
\hline
\end{tabular}

\section{Outcomes in Patients Undergoing Cystectomy}

One hundred patients $(21 \%)$ enrolled onto the six trials ultimately underwent cystectomy; 62\% underwent immediate cystectomy for incomplete response to induction chemotherapy and RT, $36 \%$ underwent salvage cystectomy after CMT for recurrences detected in follow-up, and $2 \%$ underwent cystectomy for other causes. The absolute incidence of nodal recurrence was $22 \%$ after any cystectomy $(\mathrm{n}=22), 27 \%$ after immediate cystectomy $(\mathrm{n}=17)$, and $14 \%$ after salvage cystectomy $(\mathrm{n}=5)$. Among patients undergoing cystectomy, the 5- and 10-year OS rates were $45 \%$ and $18 \%$, respectively, whereas the 5- and 10-year DSS rates were $60 \%$ and $47 \%$, respectively.

\section{Subgroup and Multivariable Analyses}

On univariable analysis, higher T stage and presence of hydronephrosis were associated with decreased DSS, and a visibly complete TURBT was associated with increased DSS, but not on multivariable analysis (Table 3). On univariable analysis, higher T stage, hydronephrosis, older age, and less than visibly complete TURBT were associated with decreased OS, but only age was associated with OS on multivariable analysis (Table 4). Visibly complete TURBT was associated with a higher CR rate to treatment in both univariable and multivariable analysis (Table 5). 


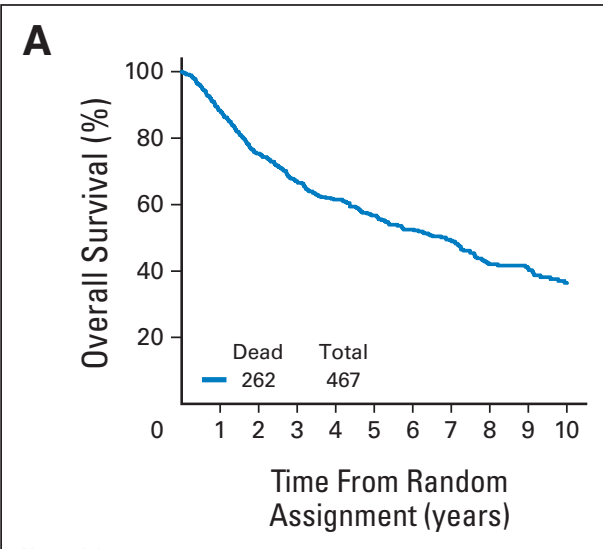

No. at risk
B

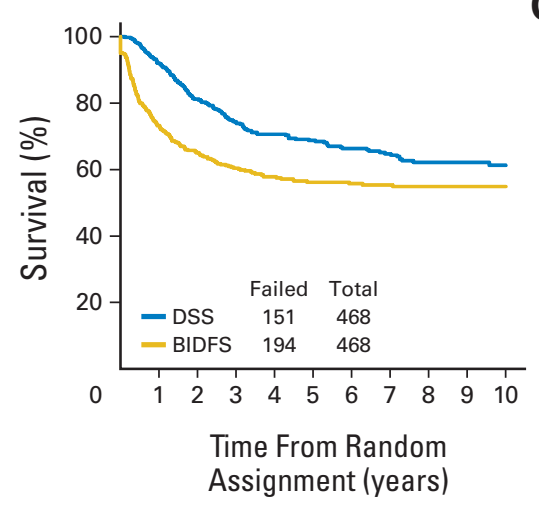

No. at risk

DSS
C

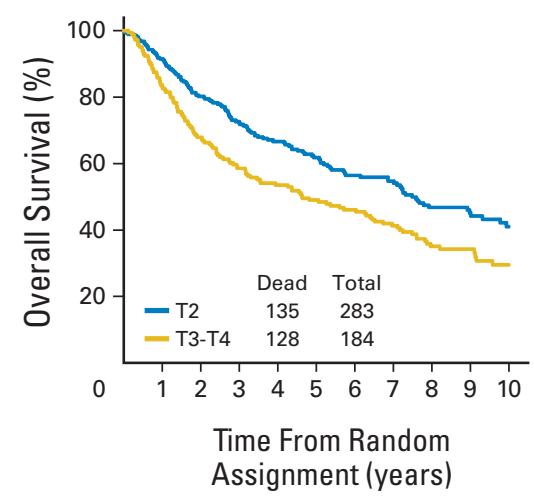

No. at risk

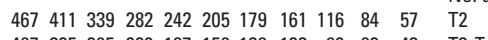
$\begin{array}{llllllllllll}467 & 325 & 265 & 220 & 187 & 156 & 138 & 123 & 90 & 62 & 43 & \text { T3-T4 }\end{array}$ $\begin{array}{lllllllllll}282 & 256 & 213 & 176 & 145 & 120 & 101 & 93 & 72 & 52 & 33\end{array}$ $\begin{array}{lllllllllll}184 & 154 & 125 & 105 & 96 & 84 & 77 & 67 & 43 & 31 & 24\end{array}$
D

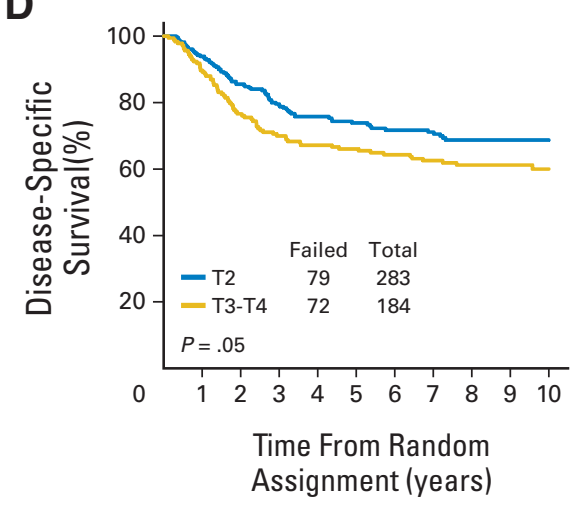

No. at risk

T2

T3-T4
E

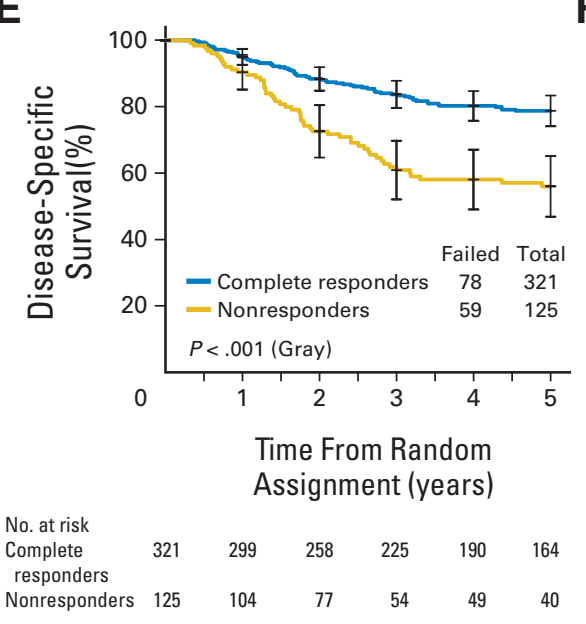

$\mathbf{F}$

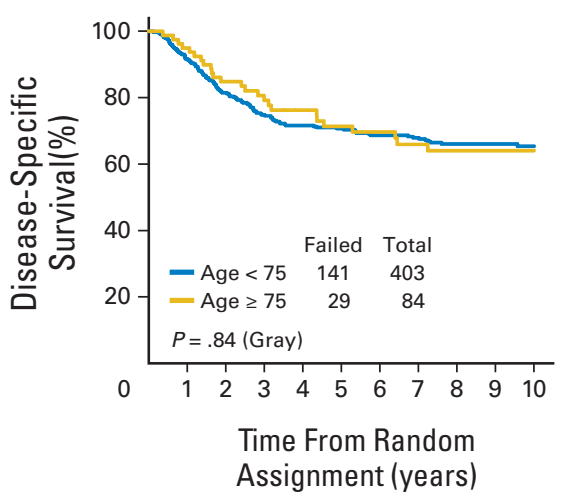

No. at risk

Age $<75$ Age $\geq 75$

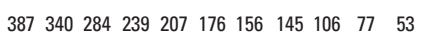
$\begin{array}{rrrrrrrrrrr}80 & 71 & 55 & 43 & 35 & 29 & 23 & 16 & 10 & 7 & 4\end{array}$

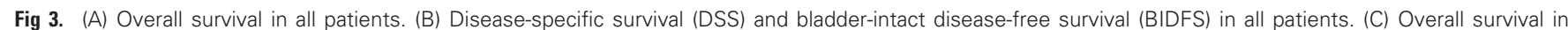

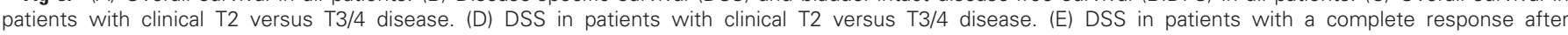

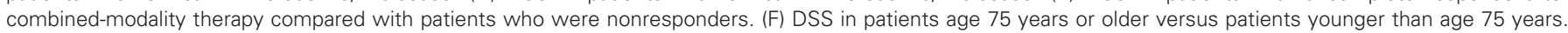

Examining outcomes by subgroups, higher clinical T stage (T2v T3/T4) was associated with decreased OS (5-year OS, 62\% v 49\%, respectively; 10 -year OS, $41 \% \vee 30 \%$, respectively; $P=.002$; Fig 3C) and DSS (5-year DSS, 74\% v 66\%, respectively; 10-year DSS, 69\% v
$60 \%$, respectively; $P=.05$; Fig 3D). Analyzing outcomes by age, elderly patients (age $\geq 75$ years) completed induction chemotherapy and RT less frequently than younger patients (age $<75$ years; 78\% $v$ $88 \%$, respectively; $P=.028$ ), but there was no difference in receiving

\begin{tabular}{|c|c|c|c|c|c|c|c|}
\hline \multirow[b]{2}{*}{ Outcome } & \multirow[b]{2}{*}{$\begin{array}{l}\text { No. of } \\
\text { Patients }\end{array}$} & \multicolumn{3}{|c|}{5 Years } & \multicolumn{3}{|c|}{10 Years } \\
\hline & & $\begin{array}{l}\text { Estimate } \\
\qquad(\%)\end{array}$ & $\begin{array}{c}95 \% \mathrm{Cl} \\
(\%)\end{array}$ & $\begin{array}{l}\text { No. of Patients } \\
\text { at Risk }\end{array}$ & $\begin{array}{l}\text { Estimate } \\
(\%)\end{array}$ & $\begin{array}{c}95 \% \mathrm{Cl} \\
(\%)\end{array}$ & $\begin{array}{c}\text { No. of Patients } \\
\text { at Risk }\end{array}$ \\
\hline Local failure, any & 212 & 43 & 39 to 48 & 148 & 48 & 43 to 53 & 39 \\
\hline Local failure, muscle invasive & 56 & 13 & 10 to 17 & 191 & 14 & 10 to 17 & 52 \\
\hline Local failure, non-muscle invasive & 156 & 31 & 27 to 36 & 162 & 36 & 32 to 41 & 44 \\
\hline Nodal recurrence & 66 & 13 & 10 to 16 & 199 & 16 & 12 to 19 & 54 \\
\hline Distant metastases & 153 & 31 & 27 to 36 & 188 & 35 & 30 to 39 & 53 \\
\hline Disease-specific survival & 150 & 71 & 67 to 75 & 205 & 65 & 61 to 70 & 57 \\
\hline Bladder-intact disease-free survival & 282 & 56 & 51 to 61 & 173 & 55 & 50 to 60 & 45 \\
\hline Overall survival & 262 & 57 & 52 to 61 & 205 & 36 & 31 to 42 & 57 \\
\hline
\end{tabular}




\begin{tabular}{|c|c|c|c|c|c|c|c|c|}
\hline \multirow[b]{2}{*}{ Variable } & \multirow[b]{2}{*}{$\begin{array}{c}\text { No. of } \\
\text { Patients }\end{array}$} & \multirow[b]{2}{*}{$\begin{array}{l}\text { No. of Bladder } \\
\text { Deaths }\end{array}$} & \multicolumn{3}{|c|}{ Univariable Regression Model } & \multicolumn{3}{|c|}{ Multiple Regression Model* } \\
\hline & & & $\begin{array}{c}\text { Hazard } \\
\text { Ratio }\end{array}$ & $95 \% \mathrm{Cl}$ & $P$ & $\begin{array}{l}\text { Hazard Ratio } \\
\text { (adjusted) }\end{array}$ & $95 \% \mathrm{Cl}$ & $P$ \\
\hline Age, years & 377 & 114 & 1.01 & 0.99 to 1.03 & .46 & 1.01 & 0.99 to 1.03 & .39 \\
\hline \multicolumn{9}{|l|}{ Sex } \\
\hline Male (RL) & 312 & 91 & 1.24 & 0.78 to 1.96 & .36 & 1.31 & 0.81 to 2.11 & .27 \\
\hline Female & 65 & 23 & & & & & & \\
\hline \multicolumn{9}{|l|}{ T stage } \\
\hline $\mathrm{T} 2(\mathrm{RL})$ & 232 & 59 & 1.73 & 1.20 to 2.49 & .0035 & 1.45 & 0.97 to 2.18 & .071 \\
\hline T3/T4a & 145 & 55 & & & & & & \\
\hline \multicolumn{9}{|l|}{ Histology } \\
\hline Urothelial (RL) & 356 & 108 & 1.03 & 0.45 to 2.35 & .95 & 0.85 & 0.39 to 1.86 & .690 \\
\hline Squamous/adenocarcinoma/other & 21 & 6 & & & & & & \\
\hline \multicolumn{9}{|l|}{ Tumor grade } \\
\hline Low grade $(R L)$ & 55 & 20 & 0.75 & 0.48 to 1.26 & .30 & 0.57 & 0.53 to 1.44 & .59 \\
\hline High grade & 322 & 94 & & & & & & \\
\hline \multicolumn{9}{|l|}{ Presence of hydronephrosis } \\
\hline No (RL) & 337 & 96 & 1.97 & 1.19 to 3.24 & .0081 & 1.70 & 0.99 to 2.91 & .052 \\
\hline Yes & 40 & 18 & & & & & & \\
\hline \multicolumn{9}{|l|}{ Visibly complete TURBT } \\
\hline Yes (RL) & 331 & 95 & 1.79 & 1.07 to 2.97 & .025 & 1.50 & 0.88 to 2.56 & .14 \\
\hline No & 46 & 19 & & & & & & \\
\hline $\begin{array}{l}\text { NOTE. Of } 468 \text { patients in the study, } \\
\text { Abbreviations: RL, reference level; } T \\
\text { "Multiple regression model, adjusted } \\
\text { tInstitutional grade. }\end{array}$ & $\begin{array}{l}\text { analysis e } \\
\mathrm{BT} \text {, transur } \\
\text { all factors }\end{array}$ & $\begin{array}{l}\text { ded } 91 \text { patients } \\
\text { al resection of bla }\end{array}$ & $\begin{array}{l}\text { missing } \\
\text { er tumor. }\end{array}$ & on tumor grac & nd/or v & er the TURBT & visibly comp & not. \\
\hline
\end{tabular}

more than 60 Gy of RT by age group ( $67 \% v 71 \%$, respectively; $P=.42)$. Between elderly and younger patients, there was no difference in CR rates (72\% $v 73 \%$, respectively; $P=.78$ ) and DSS rates (5-year DSS, 71\% $v 70 \%$, respectively; 10 -year DSS, $64 \% \vee 65 \%$, respectively; $P=.84$; Fig 3F).
Among elderly survivors at 5 years $(\mathrm{n}=22), 76 \%$ had an intact bladder, which was not significantly different than younger patients $(81 \% ; P=$ $.55)$. Bladder cancer was the cause of death at 5 years in $16 \%$ of elderly patients compared with $26 \%$ of younger patients $(P=.29)$.

\begin{tabular}{|c|c|c|c|c|c|c|c|c|}
\hline \multirow[b]{2}{*}{ Variable } & \multirow[b]{2}{*}{$\begin{array}{l}\text { No. of } \\
\text { Patients }\end{array}$} & \multirow[b]{2}{*}{$\begin{array}{l}\text { No. of } \\
\text { Deaths }\end{array}$} & \multicolumn{3}{|c|}{ Univariable Regression Model } & \multicolumn{3}{|c|}{ Multiple Regression Model* } \\
\hline & & & $\begin{array}{c}\text { Hazard } \\
\text { Ratio }\end{array}$ & $95 \% \mathrm{Cl}$ & $P$ & $\begin{array}{l}\text { Hazard Ratio } \\
\text { (adjusted) }\end{array}$ & $95 \% \mathrm{Cl}$ & $P$ \\
\hline Age, years & 377 & 206 & 1.02 & 1.01 to 1.04 & .0048 & 1.02 & 1.01 to 1.04 & .0056 \\
\hline \multicolumn{9}{|l|}{ Sex } \\
\hline Male (RL) & 312 & 172 & 0.95 & 0.66 to 1.38 & .80 & 1.03 & 0.71 to 1.50 & .87 \\
\hline T2 (RL) & 232 & 106 & 1.45 & 1.10 to 1.92 & .0078 & 1.29 & 0.96 to 1.74 & .10 \\
\hline T3/T4a & 145 & 100 & & & & & & \\
\hline \multicolumn{9}{|l|}{ Histology } \\
\hline Urothelial (RL) & 356 & 191 & 1.40 & 0.83 to 2.37 & .21 & 1.23 & 0.72 to 2.10 & .45 \\
\hline Squamous/adenocarcinoma/other & 21 & 15 & & & & & & \\
\hline \multicolumn{9}{|l|}{ Tumor grade† } \\
\hline \multicolumn{9}{|l|}{ Presence of hydronephrosis } \\
\hline Yes & 40 & 27 & & & & & & \\
\hline \multicolumn{9}{|l|}{ Visibly complete TURBT } \\
\hline Yes (RL) & 331 & 172 & 1.38 & 0.95 to 2.00 & .09 & 1.21 & 0.82 to 1.79 & .33 \\
\hline No & 46 & 34 & & & & & & \\
\hline
\end{tabular}




\begin{tabular}{|c|c|c|c|c|c|c|c|c|}
\hline \multirow[b]{2}{*}{ Variable } & \multirow[b]{2}{*}{$\begin{array}{l}\text { No. of } \\
\text { Patients }\end{array}$} & \multirow[b]{2}{*}{$\begin{array}{l}\text { No. of Complete } \\
\text { Responses }\end{array}$} & \multicolumn{3}{|c|}{ Univariable Regression Model } & \multicolumn{3}{|c|}{ Multiple Regression Model* } \\
\hline & & & $\begin{array}{l}\text { Odds } \\
\text { Ratio }\end{array}$ & $95 \% \mathrm{Cl}$ & $P$ & $\begin{array}{l}\text { Odds Ratio } \\
\text { (adjusted) }\end{array}$ & $95 \% \mathrm{Cl}$ & $P$ \\
\hline Age, years & 361 & 257 & 1.02 & 0.99 to 1.04 & .105 & 1.02 & 0.99 to 1.04 & .11 \\
\hline \multicolumn{9}{|l|}{ Sex } \\
\hline Male (RL) & 300 & 217 & 0.73 & 0.41 to 1.31 & .29 & 0.75 & 0.41 to 1.38 & .36 \\
\hline Female & 61 & 40 & & & & & & \\
\hline \multicolumn{9}{|l|}{ T stage } \\
\hline $\mathrm{T} 2$ (RL) & 226 & 167 & 0.71 & 0.44 to 1.12 & .14 & 0.81 & 0.48 to 1.37 & .44 \\
\hline Т3/Т4а & 135 & 90 & & & & & & \\
\hline \multicolumn{9}{|l|}{ Histology } \\
\hline Urothelial (RL) & 341 & 241 & 1.66 & 0.54 to 5.08 & .38 & 1.98 & 0.63 to 6.23 & .24 \\
\hline Squamous/adenocarcinoma/other & 20 & 16 & & & & & & \\
\hline \multicolumn{9}{|l|}{ Tumor grade $\dagger$} \\
\hline Low grade (RL) & 54 & 36 & 1.29 & 0.69 to 2.38 & .43 & 1.24 & 0.65 to 2.35 & .51 \\
\hline High grade & 307 & 221 & & & & & & \\
\hline \multicolumn{9}{|l|}{ Presence of hydronephrosis } \\
\hline No (RL) & 322 & 232 & 0.69 & 0.34 to 1.39 & .30 & 0.83 & 0.39 to 1.80 & .64 \\
\hline Yes & 39 & 25 & & & & & & \\
\hline \multicolumn{9}{|l|}{ Visibly complete TURBT } \\
\hline Yes (RL) & 318 & 233 & 0.46 & 0.24 to 0.88 & .020 & 0.49 & 0.25 to 0.96 & .04 \\
\hline No & 43 & 24 & & & & & & \\
\hline $\begin{array}{l}\text { NOTE. Of } 468 \text { patients in the study, } \\
\text { and } 16 \text { patients without assessment } \\
\text { Abbreviations: RL, reference level; T } \\
\text { "Multiple regression model, adjustec } \\
\text { tInstitutional grade. }\end{array}$ & $\begin{array}{l}\text { analysis ex } \\
\text { sponse aft } \\
T \text {, transure } \\
\text { all factors. }\end{array}$ & $\begin{array}{l}\text { ed } 91 \text { patients with } \\
\text { hemoradiation. } \\
\text { I resection of bladd }\end{array}$ & $\begin{array}{l}\text { sing da } \\
\text { umor. }\end{array}$ & tumor grade & or wh & r the TURBT & visibly comple & \\
\hline
\end{tabular}

\section{DISCUSSION}

In this RTOG pooled analysis of long-term outcomes of selective bladder-preserving CMT in the multi-institutional setting, we demonstrate that this treatment approach results in low rates of invasive tumor recurrence (10-year invasive LF, 14\%) and high long-term DSS (5- and 10-year DSS, 71\% and 65\%, respectively) and OS (5- and 10 -year OS, $57 \%$ and $36 \%$, respectively), with $80 \%$ of patients retaining an intact bladder at 5 years. This study provides a unique insight into the outcomes of the bladder-preserving CMT approach over two decades in the multi-institutional setting with one of the largest cohorts of patients reported to date.

Our findings build on decades of experience with bladderpreservation approaches involving more than 1,000 patients treated in single institutions and cooperative groups in North America and Europe. The MGH and Erlangen series demonstrated 5-year OS rates of $52 \%$ and $45 \%, 10$-year OS rates of $35 \%$ and $29 \%,{ }^{9-12} 5$-year DSS rates of $63 \%$ and $56 \%$, and 10 -year DSS rates of $59 \%$ and $42 \%$, respectively, which are comparable to the results observed in this pooled analysis.

Although our study demonstrates excellent long-term cancer control, bladder-preserving CMT has not been routinely adopted because of concerns including long-term toxicity from radiation to the bladder and the feasibility and curability of salvage cystectomy in patients with local recurrences. First, although we do not report toxicity outcomes in this study, previously reported long-term toxicity data from patients enrolled onto RTOG studies ${ }^{13}$ and studies from other centers $^{22-25}$ have demonstrated a low risk of toxicity and good quality-of-life outcomes with preservation of a functional bladder.
Second, we found in this study that patients who ultimately required salvage cystectomy for nonresponse to CMT or recurrent disease still had a 5 -year DSS of $60 \%$ and 10 -year DSS of $47 \%$. Combined with recent data showing that the risk of complications from RC after CMT ( $16 \%$ incidence of major complications within 90 days) is acceptable compared with upfront $\mathrm{RC},{ }^{26}$ bladder-preserving CMT is a reasonable alternative treatment in selected patients.

Although there are no randomized studies comparing RC with bladder-preserving CMT and any direct comparison is difficult because of selection bias and confounding from discordance between clinical and pathologic staging, ${ }^{27}$ the 5- and 10-year OS and DSS rates in contemporary RC series of clinically staged patients with T2-4a MIBC are comparable to those seen in this study and other bladderpreserving studies. ${ }^{1-8}$ Although organ-conserving approaches with CMT have become adopted for anal cancer and head and neck cancers, the general acceptance and adoption of bladder-preserving therapy for MIBC have been met with resistance. It is unlikely that a randomized trial comparing $\mathrm{RC}$ versus bladder-preserving CMT will be completed, given the recent failure of the United Kingdom Selective Bladder Preservation Against Radical Excision trial to accrue. ${ }^{28}$ Thus, the results of this pooled analysis provide a unique insight into the efficacy of the RTOG bladder-preserving CMT approach and serve as a useful benchmark for future bladder-preserving approaches.

Regarding patterns of failure observed in this pooled analysis, the majority of LFs were non-muscle invasive. With more than 7 years of follow-up in survivors, the majority of muscle-invasive and metastatic failures occurred within 5 years, and such recurrences beyond 5 years were uncommon. However, the 10-year nonmuscle-invasive recurrence rate was $36 \%$, underscoring the 
importance of close surveillance with routine cystoscopy and treatment if indicated after CMT. Such long-term outcome data from these RTOG studies are important in establishing selective bladder-preserving CMT as a safe and effective alternative to cystectomy. ${ }^{29}$ Although concurrent chemotherapy was used in all of these RTOG studies and many of the studies included neoadjuvant or adjuvant cisplatin-based chemotherapy, DM remained a substantial problem (10-year estimate, 35\%), which underscores the need for continued refinement of the CMT approach by incorporating new, efficacious systemic therapies with lower toxicity profiles.

This pooled analysis allowed the comparison of outcomes by subgroups, which is of interest in a relatively rare disease. Elderly patients (age $\geq 75$ years) had similar incidences of completion of RT greater than $60 \mathrm{~Gy}$, bladder preservation, and DSS compared with younger patients, demonstrating that this potentially curative CMT should be considered for elderly patients who may not be eligible for surgery and have historically had limited treatment options. In addition, higher clinical T stage (T2 $v$ T3/4) and presence of hydronephrosis were associated with decreased DSS, whereas visibly complete TURBT was associated with increased DSS on univariable analysis; these are associations that have been observed in other large series. ${ }^{9-11}$ A visibly complete TURBT was associated with higher CR rate on both univariable and multivariable analysis, and thus, we recommend an aggressive, visibly complete TURBT when feasible. However, the inability to perform a complete TURBT should not preclude an attempt at bladder preservation, because our study demonstrates that more than $50 \%$ of patients with an incomplete TURBT can still have a postinduction CR. Finally, our study again demonstrated that hydronephrosis is associated with worse outcomes with CMT, as it is with $\mathrm{RC}$, and remains a relative contraindication to bladder-preserving CMT in RTOG studies.

As a pooled analysis, this study is not powered to compare the different CMT approaches in each trial. Given the difficulties in accruing large numbers of patients to bladder-preservation trials, randomized trials to compare CMT regimens will likely be difficult to complete. Thus, the current approach of the RTOG to systematically refine the bladder-preserving CMT approach with successive phase II trials testing new regimens will lead to the continued evolution of $\mathrm{CMT}^{30}$ Although the optimal bladder-preserving regimen continues to evolve, common contemporary approaches include the RTOG approaches of concurrent chemotherapy with cisplatin/FU or RT- sensitizing low-dose gemcitabine. Recent results from a United Kingdom bladder-preservation trial demonstrated that a regimen of concurrent FU ( $500 \mathrm{mg} / \mathrm{m}^{2}$ continuous infusion, days 1 through 5 and 16 through 20 ) and mitomycin ( $12 \mathrm{mg} / \mathrm{m}^{2}$, day 1 only) also resulted in high response rates, bladder preservation, and $\mathrm{OS}^{31}$ and provides an additional chemotherapy option for patients who are unable to tolerate platinum or gemcitabine.

In conclusion, over several decades, the RTOG has refined the CMT approach by improving patient selection, RT techniques, and chemotherapeutics. This pooled analysis of RTOG trials of bladderpreserving CMT for MIBC demonstrates long-term outcomes similar to cystectomy. Thus, bladder-preserving CMT has become a safe, tested, efficacious alternative to RC in selected patients with MIBC who desire to keep their bladders. For patients with MIBC who are noncystectomy candidates or for select patients who are motivated to keep their native bladders, bladder-preserving CMT has been recognized recently in the guidelines by the International Consultation on Urological Diseases-European Association of Urology ${ }^{29}$ and by the National Comprehensive Cancer Center Network ${ }^{32}$ as an effective alternative to RC and should be considered for these patients with MIBC. Future work will continue within the RTOG and other groups to refine the bladderpreserving approach by developing new RT sensitizers ${ }^{33}$ and identifying predictive biomarkers $^{34}$ to further improve outcomes.

\section{AUTHORS' DISCLOSURES OF POTENTIAL CONFLICTS OF INTEREST}

Disclosures provided by the authors are available with this article at www.jco.org.

\section{AUTHOR CONTRIBUTIONS}

Conception and design: Raymond H. Mak, Daniel Hunt, William U. Shipley, Jason A. Efstathiou, William J. Tester, Anthony L. Zietman Provision of study materials or patients: William J. Tester

Collection and assembly of data: Raymond H. Mak, Daniel Hunt, William J. Tester, Michael P. Hagan, Donald S. Kaufman

Data analysis and interpretation: Raymond H. Mak, Daniel Hunt, William U. Shipley, Jason A. Efstathiou, Niall M. Heney, Anthony L. Zietman

Manuscript writing: All authors

Final approval of manuscript: All authors

\section{REFERENGES}

1. Stein JP, Lieskovsky G, Cote R, et al: Radical cystectomy in the treatment of invasive bladder cancer: Long-term results in 1,054 patients. J Clin Oncol 19:666-675, 2001

2. Dalbagni G, Genega $E$, Hashibe $M$, et al: Cystectomy for bladder cancer: A contemporary series. J Urol 165:1111-1116, 2001

3. Zehnder P, Studer UE, Skinner EC, et al: Super extended versus extended pelvic lymph node dissection in patients undergoing radical cystectomy for bladder cancer: A comparative study. J Urol 186:1261-1268, 2011

4. Madersbacher S, Hochreiter W, Burkhard F, et al: Radical cystectomy for bladder cancer today: A homogeneous series without neoadjuvant therapy. J Clin Oncol 21:690-696, 2003
5. Hautmann RE, Gschwend JE, de Petriconi $\mathrm{RC}$, et al: Cystectomy for transitional cell carcinoma of the bladder: Results of a surgery only series in the neobladder era. J Urol 176:486-492, 2006

6. Grossman HB, Natale RB, Tangen CM, et al: Neoadjuvant chemotherapy plus cystectomy compared with cystectomy alone for locally advanced bladder cancer. N Engl J Med 349:859-866, 2003

7. Munro NP, Sundaram SK, Weston PM, et al: A 10-year retrospective review of a nonrandomized cohort of 458 patients undergoing radical radiotherapy or cystectomy in Yorkshire, UK. Int J Radiat Oncol Biol Phys 77:119-124, 2010

8. Kotwal S, Choudhury A, Johnston $C$, et al: Similar treatment outcomes for radical cystectomy and radical radiotherapy in invasive bladder cancer treated at a United Kingdom specialist treatment center. Int J Radiat Oncol Biol Phys 70:456-463, 2008
9. Shipley WU, Kaufman DS, Zehr E, et al: Selective bladder preservation by combined modality protocol treatment: Long-term outcomes of 190 patients with invasive bladder cancer. Urology 60: 62-67, 2002

10. Rödel C, Grabenbauer GG, Kühn R, et al: Combined-modality treatment and selective organ preservation in invasive bladder cancer: Long-term results. J Clin Oncol 20:3061-3071, 2002

11. Efstathiou JA, Spiegel DY, Shipley WU, et al: Long-term outcomes of selective bladder preservation by combined-modality therapy for invasive bladder cancer: The MGH experience. Eur Urol 61:705-711, 2012

12. Weiss $C$, Engehausen DG, Krause FS, et al: Radiochemotherapy with cisplatin and 5-fluorouracil after transurethral surgery in patients with bladder cancer. Int J Radiat Oncol Biol Phys 68:1072-1080, 2007 
13. Efstathiou JA, Bae K, Shipley WU, et al: Late pelvic toxicity after bladder-sparing therapy in patients with invasive bladder cancer: RTOG 89-03, 95-06, 97-06, 99-06. J Clin Oncol 27:4055-4061، 2009

14. Tester W, Caplan R, Heaney J, et al: Neoadjuvant combined modality program with selective organ preservation for invasive bladder cancer: Results of Radiation Therapy Oncology Group phase II trial 8802. J Clin Oncol 14:119-126, 1996

15. Shipley WU, Winter KA, Kaufman DS, et al: Phase III trial of neoadjuvant chemotherapy in patients with invasive bladder cancer treated with selective bladder preservation by combined radiation therapy and chemotherapy: Initial results of Radiation Therapy Oncology Group 89-03. J Clin Oncol 16:3576-3583, 1998

16. Kaufman DS, Winter KA, Shipley WU, et al: The initial results in muscle-invading bladder cancer of RTOG 95-06: Phase I/II trial of transurethral surgery plus radiation therapy with concurrent cisplatin and 5-fluorouracil followed by selective bladder preservation or cystectomy depending on the initial response. Oncologist 5:471-476, 2000

17. Hagan MP, Winter KA, Kaufman DS, et al: RTOG 97-06: Initial report of a phase I-II trial of selective bladder conservation using TURBT, twicedaily accelerated irradiation sensitized with cisplatin, and adjuvant MCV combination chemotherapy. Int J Radiat Oncol Biol Phys 57:665-672, 2003

18. Kaufman DS, Winter KA, Shipley WU, et al: Phase I-II RTOG study (99-06) of patients with muscle-invasive bladder cancer undergoing transurethral surgery, paclitaxel, cisplatin, and twice-daily radiotherapy followed by selective bladder preservation or radical cystectomy and adjuvant chemotherapy. Urology 73:833-837, 2009
19. Mitin T, Hunt D, Shipley WU, et al: Transurethral surgery and twice-daily radiation plus paclitaxelcisplatin or fluorouracil-cisplatin with selective bladder preservation and adjuvant chemotherapy for patients with muscle invasive bladder cancer (RTOG 0233): A randomised multicentre phase 2 trial. Lancet Oncol 14:863-872, 2013

20. Kaplan EL, Meier P: Nonparametric estimation from incomplete observations. J Am Stat Assoc 53:447-457, 1958

21. Gray RJ: A class of K-sample tests for comparing the cumulative incidence of a competing risk. Ann Stat 16:1141-1143, 1988

22. Zietman $A L$, Sacco $D$, Skowronski $U$, et al: Organ conservation in invasive bladder cancer by transurethral resection, chemotherapy and radiation: Results of a urodynamic and quality of life study on long-term survivors. J Urol 170:1772-1776, 2003

23. Henningsohn L, Steven K, Kallestrup EB, et al: Distressful symptoms and well-being after radical cystectomy and orthotopic bladder substitution compared with a matched control population. J Urol 168:168-174, 2002

24. Lagrange JL, Bascoul-Mollevi C, Geoffrois L, et al: Quality of life assessment after concurrent chemoradiation for invasive bladder cancer: Results of a multicenter prospective study (GETUG 97-015). Int J Radiat Oncol Biol Phys 79:172-178, 2011

25. Caffo O, Fellin G, Graffer U, et al: Assessment of quality of life after cystectomy or conservative therapy for patients with infiltrating bladder carcinoma: A survey by a self-administered questionnaire. Cancer 78:1089-1097, 1996

26. Eswara JR, Efstathiou JA, Heney NM, et al: Complications and long-term results of salvage cystectomy after failed bladder sparing therapy for muscle invasive bladder cancer. J Urol 187:463-468, 2012
27. Gray PJ, Fedewa SA, Shipley WU, et al: Clinical-pathologic stage discrepancy in patients with bladder cancer treated with radical cystectomy: Associations with clinical variables and survival. J Clin Oncol 31, 2013 (suppl 6; abstr 248)

28. Huddart RA, Hall E, Lewis R, et al: Life and death of SPARE (selective bladder preservation against radical excision): Reflections on why the SPARE trial closed. BJU Int 106:753-755, 2010

29. Gakis G, Efstathiou J, Lerner SP, et al: ICUDEAU International Consultation on Bladder Cancer 2012: Radical cystectomy and bladder preservation for muscle-invasive urothelial carcinoma of the bladder. Eur Urol 63:45-57, 2013

30. Shipley WU, Efstathiou JA: Radiation-based bladder preserving strategies: Radiation alone or combined with other modalities, in Soloway M, Khoury S (eds): Bladder Cancer: International Consultation on Bladder Cancer-Vienna (ed 2). Paris, France, International Consultation on Urological Diseases-European Association of Urology, 2012, pp 316-326

31. James ND, Hussain SA, Hall E, et al: Radiotherapy with or without chemotherapy in muscleinvasive bladder cancer. N Engl J Med 366: 1477-1488, 2012

32. Clark PE, Agarwal N, Biagioli MC, et al: Bladder cancer. J Natl Compr Canc Netw 11:446-475, 2013

33. Hoskin PJ, Rojas AM, Bentzen SM, et al: Radiotherapy with concurrent carbogen and nicotinamide in bladder carcinoma. J Clin Oncol 28:49124918, 2010

34. Choudhury A, Nelson LD, Teo MT, et al: MRE11 expression is predictive of cause-specific survival following radical radiotherapy for muscleinvasive bladder cancer. Cancer Res 70:7017-7026, 2010 


\section{AUTHORS' DISCLOSURES OF POTENTIAL CONFLICTS OF INTEREST}

Long-Term Outcomes in Patients With Muscle-Invasive Bladder Cancer After Selective Bladder-Preserving Combined-Modality Therapy: A Pooled Analysis of Radiation Therapy Oncology Group Protocols 8802, 8903, 9506, 9706, 9906, and 0233

The following represents disclosure information provided by authors of this manuscript. All relationships are considered compensated. Relationships are self-held unless noted. I = Immediate Family Member, Inst $=$ My Institution. For a detailed description of the disclosure categories, or for more information about ASCO's conflict of interest policy, please refer to the Author Disclosure Declaration and the Disclosures of Potential Conflicts of Interest section in Information for Contributors.

Raymond H. Mak

Consulting or Advisory Role: Celgene

Stock or Other Ownership: Boehringer Ingelheim

\section{Daniel Hunt}

Employment: Puma

Stock or Other Ownership: Puma

Research Funding: Puma

Travel, Accommodations, Expenses: Puma

\section{William U. Shipley}

Stock or Other Ownership: Pfizer

\section{Jason A. Efstathiou}

Consulting or Advisory Role: Medivation/Astellas, Bayer
William J. Tester

No relationship to disclose

Michael P. Hagan

No relationship to disclose

Donald S. Kaufman

No relationship to disclose

\section{Niall M. Heney}

No relationship to disclose

Anthony L. Zietman

No relationship to disclose 


\section{Appendix}

Table A1. Overview of RTOG Bladder-Preserving Combined-Modality Therapy Trials for Muscle-Invasive Bladder Cancer

\begin{tabular}{|c|c|c|c|c|}
\hline RTOG Protocol & Neoadjuvant Chemotherapy & ChemoRT $^{*}$ & Adjuvant Chemotherapy & No. of Eligible Patients \\
\hline 8802 & 2 cycles MCV & Cisplatin + RT & None & 90 \\
\hline \multicolumn{5}{|l|}{8903} \\
\hline Arm 1 & 2 cycles MCV & Cisplatin + RT & None & 61 \\
\hline Arm 2 & None & Cisplatin + RT & None & 62 \\
\hline 9506 & None & Cisplatin/FU + RT & None & 34 \\
\hline 9706 & None & Cisplatin/FU + BID RT & MCV & 47 \\
\hline 9906 & None & Cisplatin/paclitaxel + BID RT & Cisplatin/gemcitabine & 81 \\
\hline \multicolumn{5}{|l|}{0233} \\
\hline Arm 1 & None & Cisplatin/paclitaxel + BID RT & Cisplatin/paclitaxel/gemcitabine & 46 \\
\hline Arm 2 & None & Cisplatin/FU + BID RT & & 47 \\
\hline
\end{tabular}

\begin{tabular}{|c|c|c|c|c|c|c|c|c|c|c|c|c|c|c|}
\hline \multirow[b]{2}{*}{ Trial } & \multirow[b]{2}{*}{$\begin{array}{l}\text { No. of } \\
\text { Patients }\end{array}$} & \multirow[b]{2}{*}{$\begin{array}{c}\text { Follow-Up } \\
\text { (years) }\end{array}$} & \multicolumn{2}{|c|}{ Any LF (\%) } & \multicolumn{2}{|c|}{ MI-LF (\%) } & \multicolumn{2}{|c|}{ NMI-LF (\%) } & \multicolumn{2}{|c|}{ DM (\%) } & \multicolumn{2}{|c|}{ OS (\%) } & \multicolumn{2}{|c|}{ DSS (\%) } \\
\hline & & & $\begin{array}{c}5 \\
\text { Years }\end{array}$ & $\begin{array}{l}10 \\
\text { Years }\end{array}$ & $\begin{array}{c}5 \\
\text { Years }\end{array}$ & $\begin{array}{c}10 \\
\text { Years }\end{array}$ & $\begin{array}{c}5 \\
\text { Years }\end{array}$ & $\begin{array}{l}10 \\
\text { Years }\end{array}$ & $\begin{array}{c}5 \\
\text { Years }\end{array}$ & $\begin{array}{l}10 \\
\text { Years }\end{array}$ & $\begin{array}{c}5 \\
\text { Years }\end{array}$ & $\begin{array}{l}10 \\
\text { Years }\end{array}$ & $\begin{array}{c}5 \\
\text { Years }\end{array}$ & $\begin{array}{l}10 \\
\text { Years }\end{array}$ \\
\hline RTOG 8802 & 90 & 5.20 & 39 & 53 & 10 & 10 & 28 & 39 & 25 & 31 & 57 & 37 & 71 & 62 \\
\hline \multicolumn{15}{|l|}{ RTOG 8903} \\
\hline Arm 1 & 61 & 5 & 40 & 40 & 19 & 19 & 19 & 19 & 32 & 32 & 47 & 20 & 68 & 63 \\
\hline Arm 2 & 62 & 6 & 48 & 48 & 6 & 6 & 41 & 41 & 40 & 46 & 48 & 29 & 66 & 63 \\
\hline RTOG 9506 & 34 & 6.8 & 42 & 42 & 9 & 9 & 32 & 32 & 35 & 35 & 53 & 38 & 62 & 62 \\
\hline RTOG 9706 & 47 & 6.2 & 48 & 55 & 16 & 16 & 32 & 37 & 38 & 43 & 57 & 36 & 64 & 53 \\
\hline RTOG 9906 & 81 & 6.1 & 48 & 59 & 14 & 17 & 35 & 45 & 34 & 35 & 58 & 48 & 74 & 71 \\
\hline \multicolumn{15}{|l|}{ RTOG 0233} \\
\hline Arm 1 & 46 & 3.1 & 48 & & 11 & & 40 & & 29 & & 66 & & 70 & \\
\hline Arm 2 & 47 & 2.9 & 53 & & 28 & & 27 & & 21 & & 74 & & 89 & \\
\hline
\end{tabular}

